\title{
Associations between structures and resources of primary care at the district level and health outcomes: a case study of diabetes mellitus care in Thailand [Erratum]
}

Komwong D, Sriratanaban J. Risk Manag Healthc Policy.

2018;11:199-208.

Page 204, Table 5 was incorrect. The correct table is included

below:

Table 5 The association of structures and resources of primary care and age-standardized DM hospitalization ratio ${ }^{\mathrm{a}}(\mathrm{n}=838$ districts)

\begin{tabular}{|c|c|c|c|c|c|c|c|c|}
\hline \multirow{3}{*}{$\begin{array}{l}\text { Primary care } \\
\text { structure and } \\
\text { resources }\end{array}$} & \multirow{2}{*}{\multicolumn{2}{|c|}{$\begin{array}{l}\text { Overall DM- } \\
\text { hospitalization }\end{array}$}} & \multicolumn{6}{|c|}{ Subgroup of DM-hospitalization } \\
\hline & & & \multicolumn{2}{|l|}{$\begin{array}{l}\text { Uncontrolled } \\
\text { diabetes }\end{array}$} & \multicolumn{2}{|l|}{$\begin{array}{l}\text { Short-term } \\
\text { complications }\end{array}$} & \multicolumn{2}{|l|}{$\begin{array}{l}\text { Long-term } \\
\text { complications }\end{array}$} \\
\hline & $\begin{array}{l}\text { Coefficient } \\
\text { (SE) }\end{array}$ & $95 \% \mathrm{Cl}$ & $\begin{array}{l}\text { Coefficient } \\
\text { (SE) }\end{array}$ & $95 \% \mathrm{Cl}$ & $\begin{array}{l}\text { Coefficient } \\
\text { (SE) }\end{array}$ & $95 \% \mathrm{Cl}$ & $\begin{array}{l}\text { Coefficient } \\
\text { (SE) }\end{array}$ & $95 \% \mathrm{Cl}$ \\
\hline $\mathrm{HH} \mathrm{I}^{\mathrm{b}}$ & $0.003^{c}(0.00 I)$ & $\begin{array}{l}0.000 \\
0.006 \\
\end{array}$ & $0.005^{c}(0.002)$ & $\begin{array}{l}0.001 \\
0.010\end{array}$ & $0.001(0.002)$ & $\begin{array}{l}-0.003 \\
0.005\end{array}$ & $-0.002(0.003)$ & $\begin{array}{l}-0.007 \\
0.003\end{array}$ \\
\hline $\begin{array}{l}\text { Public providers/ } \\
100 \mathrm{~km}^{2}\end{array}$ & $-0.835(0.538)$ & \begin{tabular}{|l|}
-1.889 \\
0.219 \\
\end{tabular} & $-0.610(0.813)$ & $\begin{array}{l}2.205 \\
0.984 \\
\end{array}$ & $-2.688^{c}(0.763)$ & $\begin{array}{l}-4.183 \\
-1.192 \\
\end{array}$ & $2.584^{c}(0.95 I)$ & $\begin{array}{l}0.719 \\
4.449 \\
\end{array}$ \\
\hline Bed supply & $-0.023^{c}(0.01 \mathrm{I})$ & \begin{tabular}{|l|}
-0.045 \\
-0.001 \\
\end{tabular} & $-0.032^{c}(0.017)$ & $\begin{array}{l}-0.064 \\
-0.001\end{array}$ & $0.027(0.016)$ & $\begin{array}{l}-0.004 \\
0.057 \\
\end{array}$ & $-0.007(0.023)$ & $\begin{array}{l}-0.052 \\
0.037 \\
\end{array}$ \\
\hline $\begin{array}{l}\text { Achieving the sustainable } \\
\text { accreditation program }\end{array}$ & $1.007(2.129)$ & \begin{tabular}{|l|}
-3.166 \\
5.180 \\
\end{tabular} & $0.467(3.156)$ & $\begin{array}{l}-5.718 \\
6.653 \\
\end{array}$ & $2.180(3.158)$ & $\begin{array}{l}-4.009 \\
8.369 \\
\end{array}$ & $0.021(5.690)$ & $\begin{array}{l}-11.132 \\
11.174\end{array}$ \\
\hline Financial crisis $^{d}$ & $1.165(3.309)$ & \begin{tabular}{|l|}
-5.321 \\
7.652 \\
\end{tabular} & $0.994(4.68 \mathrm{I})$ & $\begin{array}{l}-8.181 \\
10.169\end{array}$ & $\mathrm{I} .440(5.39 \mathrm{I})$ & $\begin{array}{l}-9.126 \\
12.006\end{array}$ & $2.601(9.229)$ & $\begin{array}{l}-15.487 \\
20.689\end{array}$ \\
\hline $\begin{array}{l}\text { District located in } \\
\text { rural area }\end{array}$ & 19.6 I8c (4.206) & \begin{tabular}{|l|}
11.374 \\
$27.86 \mid$ \\
\end{tabular} & $31.045^{`}(6.322)$ & $\begin{array}{l}8.655 \\
43.436\end{array}$ & -1.3 II (5.209) & $\begin{array}{l}-11.521, \\
8.898\end{array}$ & $20.453^{c}(8.408)$ & $\begin{array}{l}3.995 \\
36.956 \\
\end{array}$ \\
\hline Physician/ $10^{4}$ pop & $-1.350^{c}(0.674)$ & \begin{tabular}{|l|}
$-2.67 I$ \\
-0.028 \\
\end{tabular} & $-3.152^{c}(0.987)$ & $\begin{array}{l}-5.087 \\
-1.218 \\
\end{array}$ & $1.673(1.022)$ & $\begin{array}{l}-0.331 \\
3.676\end{array}$ & $-1.14 \mid(2.15 I)$ & $\begin{array}{l}-5.357 \\
3.074 \\
\end{array}$ \\
\hline Nurse/I $0^{4}$ pop & $-0.155(0.180)$ & \begin{tabular}{|l|}
-0.507 \\
0.198 \\
\end{tabular} & $-0.099(0.366)$ & $\begin{array}{l}-0.817 \\
0.618\end{array}$ & $-0.488(0.255)$ & $\begin{array}{l}-0.988 \\
0.012\end{array}$ & $0.304(0.574)$ & $\begin{array}{l}-0.821 \\
1.429\end{array}$ \\
\hline Pharmacist/ $10^{4}$ pop & $-3.834(3.233)$ & \begin{tabular}{|l}
-10.171 \\
2.503 \\
\end{tabular} & $-3.080(5.610)$ & $\begin{array}{l}-14.074, \\
7.916\end{array}$ & $-3.286(3.143)$ & $\begin{array}{l}-9.446 \\
2.874 \\
\end{array}$ & $-8.869(4.590)$ & $\begin{array}{l}-17.864 \\
0.127 \\
\end{array}$ \\
\hline $\begin{array}{l}\text { Other health } \\
\text { professionals } / 10^{4} \text { pop }\end{array}$ & $0.621(0.446)$ & \begin{tabular}{|l|}
-0.254 \\
.496 \\
\end{tabular} & $0.519(0.528)$ & $\begin{array}{l}-0.516 \\
1.553\end{array}$ & $0.789(0.5 \mathrm{II})$ & $\begin{array}{l}-0.212 \\
1.790\end{array}$ & $\mathrm{I} . \mathrm{I} 85(0.88 \mathrm{I})$ & $\begin{array}{l}-0.541, \\
2.912 \\
\end{array}$ \\
\hline $\begin{array}{l}\text { Family medicine } \\
\text { physician } / 10^{4} \text { pop }\end{array}$ & $2.535(5.049)$ & \begin{tabular}{|l}
$-7.36 \mid$ \\
$|2.43|$
\end{tabular} & $1.553(6.598)$ & $\begin{array}{l}-11.378 \\
14.485\end{array}$ & $-5.782(6.964)$ & $\begin{array}{l}-19.493 \\
7.929\end{array}$ & $23.638(20.589)$ & $\begin{array}{l}-16.715, \\
63.991\end{array}$ \\
\hline
\end{tabular}

Notes: ${ }^{a}$ Adjusted for district population characteristics, \% of population aged $>60$ years, DM prevalence and DM patient characteristics, $\%$ of patients aged $>60$ years,

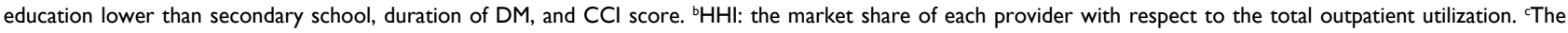
numbers in bold indicate $P<0.05$. ${ }^{d}$ The district had financial risk scoring of 7 for at least 6 of 12 quarters (between the previous two fiscal years and the current fiscal year). eOther health professionals include public health technical officers, public health officers, dental assistants, and pharmacy technicians.

Abbreviations: DM, diabetes mellitus; HHI, Herfindahl-Hirschman Index; pop, population; SE, standard error; $\mathrm{CCl}$, Charlson Comorbidity Index.

Risk Management and Healthcare Policy

Dovepress

\section{Publish your work in this journal}

Risk Management and Healthcare Policy is an international, peer-reviewed, open access journal focusing on all aspects of public health, policy, and preventative measures to promote good health and improve morbidity and mortality in the population. The journal welcomes submitted papers covering original research, basic science, clinical and epidemiological studies, reviews and evaluations, guidelines, expert opinion and commentary, case reports and extended reports. The manuscript management system is completely online and includes a very quick and fair peerreview system, which is all easy to use. Visit http://www.dovepress.com/ testimonials.php to read real quotes from published authors.

Submit your manuscript here: https://www.dovepress.com/risk-management-and-healthcare-policy-journal

submit your manuscript

Dovepress 